\title{
Application of the Donabedian Quality-of-Care Model to New York State Direct Support Professional Core Competencies: How Structure, Process, and Outcomes Impact Disability Services
}

Johanna LoPorto, $\mathrm{PhD}$

Walden University, Minneapolis, Minnesota, United States

(iD) https://orcid.org/0000-0002-3068-3418

Contact: johannaloporto@gmail.com

\begin{abstract}
Direct support professionals (DSPs) are responsible for the daily supervision and care of people diagnosed with intellectual and developmental disabilities (IDDs) living in community residential group homes. In New York State, these DSPs are trained within the Office for People With Developmental Disabilities DSPs core competencies; a set of ethical, technical, and cognitive training geared to the individual care of each person as per their specific needs. This qualitative case study was to understand how DSPs perceived the implementation of the core competencies after being trained and under the direction of their supervisors. Using the Donabedian's quality-of-care conceptual framework, this study explored what DSPs perceived to be necessary in strengthening the effectiveness of the New York State DSP competencies within organizational policies (structure) with the DSPs knowledge, skills, and, attitudes (processes) to the quality-of-life (outcomes). Data were collected through face-to-face interviews with 12 DSPs and supervisors. Data were inductively coded then subject to Braun and Clarke's thematic analysis procedure. Findings revealed that DSPs and supervisors implemented the core competencies inconsistently because of organizational perceptions and experiences. Implications for social change in this study includes recommendations to the National Alliance of DSPs to add a practicum component to the core competencies training that may benefit people living in community residential group homes diagnosed with IDDs through hands-on-approach training that would allow full implementation of the DSP core competencies in various everyday, real-life situations.
\end{abstract}

Keywords: direct support professionals, developmental disabilities, competencies

Date Submitted: February 10, 2020 | Date Published: May 8, 2020

\section{Recommended Citation}

LoPorto, J. (2020). Application of the Donabedian quality-of-care model to New York State direct support professional core competencies: How structure, process, and outcomes impact disability services. Journal of Social Change, 12, 40-70. https://doi.org/10.5590/JOSC.2020.12.1.05 
LoPorto, 2020

\section{Introduction}

People living in New York State residential group homes rely on their staff to provide 24-hr care and services as their intellectual or developmental disabilities (IDDs) do not allow them to care for themselves (Hewitt \& Lakin, 2001). These group homes provide people diagnosed with IDD rehabilitation services so each person can successfully live, work, and learn within the community. As people with IDDs continue to transition to community group homes, the need for workers to provide support care and services increase. Direct support professionals (DSPs) provide these support services with the primary function of providing personal assistance, supervision, behavioral management, and medication administration $24 \mathrm{hr}$ per day (Hewitt et al., 2004). DSPs work in several settings that include home-health agencies, hospitals, individualized residential alternatives, supportive apartments, and home-community-based service facilities.

The need for DSPs is projected to grow from 369,580 in 2010 to 505,060 by the end of 2020 (Paraprofessional Health Institute, 2013). The Office for People With Development Disabilities ([OPWDD], 2013) emphasized that nonprofit organizations providing services to people with IDD experience continuous high turnovers because of job-related stress, burnout, work demands, and low organizational morale. As a result, the care for the people in these organizations become compromised due to the increased likelihood of client abuse, neglect, and overall inadequate care and services (Hewitt \& Lakin, 2001; Hewitt \& Larson, 2007). As such, OPWDD created a competencies curriculum, that provides administrative support, structure, and oversight for the implementation of a professional development program. This program is composed of seven goals, 23 standardized competencies, and 60 identified skill sets (National Alliance for Direct Support Professionals, 2011; see Appendix).

This policy aims to provide all DSPs the education and training of standardized technical, cognitive, and ethical core competencies. The problem identification in this study, however, is that DSP competencies training and education have been developed, but it is not known how those DSP competencies are being implemented and practiced in IDD provider organizations. The goal of this qualitative research was to explore DSPs perception and experience in implementing the competencies within their organizations.

\section{Background}

The need for disabilities policy change came to the forefront when, in 1972, emerging journalist Geraldo Rivera secretly entered Building 6 of the Willowbrook School in Staten Island, New York, and, with a hidden camera, captured live video footage of the school's overcrowding; staff's dehumanizing practices; dangerous, dirty conditions of the institution; and the abuse of residents (Rothman \& Rothman, 1984).

Rivera's news coverage resulted in public outrage with advocacy groups filing a class action suit against New York. On April 30, 1975, Judge Orin Judd signed the Willowbrook consent decree, ending a 3-year legal battle to improve conditions for the mentally disabled. New York State was mandated to spend \$2 million creating small community housing and group homes providing all necessary living provisions such as food, clothing, clean and safe environments, medical and therapeutic services, programming, and education. The consent decree also mandated community integration to ultimately prepare each resident for life in the community (Rothman \& Rothman, 1984). Through the consent decree, people with IDD now transitioned from institutions to community residences. 


\section{Donabedian Quality-of-Care Model}

Extant literature on people living in these community residences demonstrates the need of operational processes that maximizes the quality of care (QOC) for residents. Donabedian's (1980) QOC model identifies three domains relevant to high-quality client care: structure, process, and outcome. Donabedian's model refers to the environment and the resources necessary to provide services that include facilities, equipment, staff, and monetary resources. The process describes the techniques and practices implemented to provide care, while outcomes are the end results realized by the recipient. According to Donabedian, an established structure is a prerequisite to effective process, and effective processes are a prerequisite for high-quality outcomes. Other researchers also used Donabedian's model to identify QOC. In 1990, the U.S. Institute of Medicine published a now widely used definition of quality care: "quality of care is the degree to which health services for individuals and populations increase the likelihood of desired health outcomes and are consistent with professional knowledge" (Lohr \& Schroeder, 1990, p. 21), expanding on Donabedian's QOC model. Camilleri and O'Callaghan (1998) developed eight attributes for their QOC model that included professionalism, technical care, service personalization, cost, environment, client amenities, accessibility, and catering. Jabnoun and Chaker (2003) used 10 dimensions for their QOC model in the medical sector that included tangibles, accessibility, understanding, courtesy, reliability, security, credibility, responsiveness, communication, and competence. In that same year, Lawthers and colleagues (2003) developed a QOC framework concluding that high QOC for people with disabilities required a link between a medical and social QOC model. All research points to the relationship between organizational characteristics, staff performance to the QOC and client outcomes.

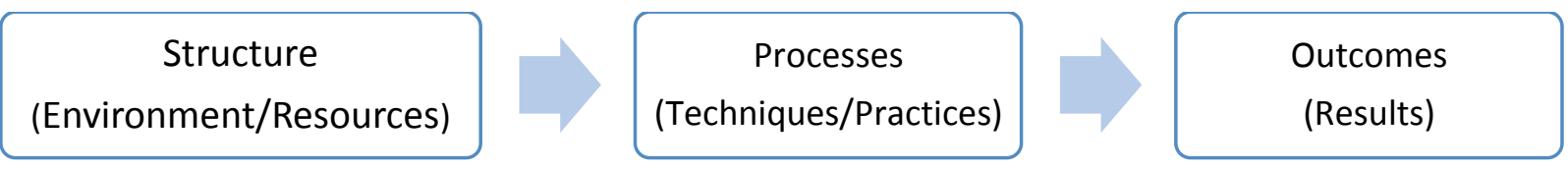

Figure 1. Donabedian Quality-of-Care Model. Adapted from p. 113 of Ranji, S. R., Kanaka, S., Posley, K. A., Lewis, R., Sundaram, V., Galvin, C., \& Winston, L. G. (2007). Closing the quality gap: A critical analysis of quality improvement strategies (Vol. 6: Prevention of healthcare-associated infections). Agency for Healthcare Research and Quality.

\section{The Role of the DSP}

Following the Willowbrook consent degree in 1975, Public Law 97-35, entitled the Medicaid Home and Community-Based Services Wavier, went into effect in 1981. This law provided a stimulus for community services and family supports, and a reduction of dependence on institutional care. It is now the principle source of funding for in-home services, comprising $70 \%$ of the funding in the United States (Rizzolo et al., 2013). The waiver allows for people living with IDD to live in community residential group homes through their life span. Thus, the DSP role began to shift from that of primary caregiver assisting the person with activities of daily living to that of a qualified professional requiring training in community supports, skill development, medication administration, behavior management, and crisis intervention (Hewitt, 1998; Hewitt \& Lakin, 2001; Hewitt et al., 2004; Larson \& Hewitt, 2005, 2012). 
LoPorto, 2020

\section{The Role of Supervisors}

Leadership behavior, disposition, and attitude plays a significant role in the satisfaction, commitment, and morale of employees (Berson \& Linton, 2005; Chun et al., 2012; Godshalk \& Sosik, 2000) The concept of leadership carries various definitions and linked to a variety of different roles (Cameron, 2011). Tuckey et al. (2012) defined leadership as having the ability to influence others, whereas Cameron (2011) added that leadership also includes having the ability to influence organizational change. Maynard and colleagues (2012) and McDermott and colleagues (2011), on the other hand, described leadership as going beyond individualistic characteristics and having the ability to balance the organization by empowering its employees.

Organizational leaders oversee the functioning of the organization, whereas managers are left to oversee the daily operations of departments. Supervisors are those that define the job, provide the training, mediate the stresses, create the culture, and establish a well-functioning work environment (Hewitt et al., 2004); at the same time, they are expected to maintain working groups and ensure operational effectiveness to achieve organizational goals (Gosling \& Mintzber, 2003). These supervisors are not always equipped to fulfill the supervisory demands of their position as leadership skills, essential training, knowledge, and competencies lack (Avolio et al., 2009; Burchard et al., 1990; Hewitt et al., 2004; Thousand et al., 1986). Mansell (2006) stated that managers who hold leadership positions need to be skilled enough to influence the direction of quality supports provided by the DSPs by ensuring education and training of the DSPs (McConkey \& Collins, 2010; Hewitt et al., 2004; Hewitt \& Larson, 2012). Problems begin to exist when these managers do not possess the skills to engage in leadership compromising working relationships and organizational operations demands (Gosling \& Mintzber, 2003). Hewitt et al. (2004) provided comprehensive research when their study of 146 participants of a focus group concluded that managers must be competent to lead.

\section{Purpose and Methodology}

The purpose of this study was to explore the relationship between policy implementation and organizational cultures. The DSP competencies are implemented across all New York State disability provider organizations (Valla, 2014); nevertheless, there is a lack of research regarding how DSPs perceive the New York State DSP competencies implemented in their organizations. Managing DSP performance continues to be problematic as challenges continue to exist in New York State IDD provider organizations. DSPs now are required to implement the DSP competencies within perceived barriers such as organizational policies, procedures or practices that limit them from implementing the competencies effectively (Larson \& Hewitt, 2012). In addition, barriers may also be perceived as directives given by frontline managers that conflict with the New York State DSP competencies. Conflicting directives and policies results in job-related stress, burnout, and low organizational morale, which ultimately impacts the service and care that is being provided to the people receiving services (Hewitt \& Lakin, 2004; Konrad \& Morgan, 2006; Larson \& Hewitt, 2012).

The purpose of research using qualitative, descriptive methods were to explore DSP experiences and perceptions of the New York State DSP competencies implementation in their organization. In this study, the researcher aimed to answer the research questions on (a) how DSPs describe the effectiveness of the New York State DSP competencies in relationship to their job satisfaction and organizational morale and (b) what DSPs perceive that it would take to enhance the effectiveness of the competencies organizational practices to ultimately improve IDD care and supports.

The participants for this study came from two New York State provider organizations, one in New York City (Agency A) and other in upstate Elmira, New York (Agency B), to provide a comparative analysis on how the New York State DSP competencies were currently being implemented in two regions of New York, southern 
and central. Both agencies provided written consent to have the study conducted and after institutional review board approval, flyers were distributed to recruit participants who met the criteria for inclusion: DSPs with work experience between 2-5 years, who were above the age of 18, and who had received the OPWDD New York State DSP core competencies training. The overriding assumption was that if DSPs worked over 2 years and were trained in OPWDD's core competencies, they could recognize discrepancies in organizational policy and practice within OPWDD's competency expectations.

The researcher served as the interviewer, and the protocol used a prescribed a semistructured interview format with questions that focused on the New York State DSP initiative implementation, organizational change, and DSP competency practices. Secondary data included training transcripts, training curricula, and written organizational policies. Data were triangulated through member-checking and sharing interview transcripts with interviewees that verified their accuracy. The other method of triangulation was the comparison of secondary data with primary data collected through the interviews. The data were analyzed through the identification of emerging themes and patterns, used with a descriptive and inductive approach. After transcription, responses were analyzed word for word and sentence by sentence. Data were also aided by a qualitative data analysis software program (CAQDAS) to assist with coding to produce descriptive and interpretive reports based on themes and patterns (Stake, 1995; Yin, 2014).

With participants' signed consent, data were derived from in-depth face-to-face interviews with four DSPs and two frontline managers from each organization, a total of 12 participants for the study through purposeful sampling (Creswell, 2009; Patton, 2002). The researcher continued to sample until saturation was reached upon discovering no additional information.

Table 1 depicts the supervisors and DSPs that participated in the study. The participants that were informed in advance that the interviews would be within two face-to-face sessions, for $1 \mathrm{hr}$, and audio-recorded. All participants granted signed consents to be interviewed and audio-recorded. The participants included a total of five men and seven women. Agency A participants were one male and five females with the male being one of the frontline managers. Agency B participants included four males and two females with two males as frontline managers. The agencies carried different titles for the frontline manager position, with Agency A referring to them as program managers and Agency B calling them program supervisors. For the purpose of this study, I referred to all employees in a supervisory position as supervisor, designated with the letter " $\mathrm{M}$ " and corresponding number to indicate the participant were in a management level position. The other eight participants were employed and referred to as DSPs. All participants from both agencies were full-time employees working in residential group homes serving people with IDD.

Questions for the interview were developed to answer each of the research questions. Interviews for both agencies were completed between 30 and 40 min for each of their sessions except for Participant G. As per his request, his interview was completed during one session that lasted $40 \mathrm{~min}$. Participant $\mathrm{G}$ was unable to meet for his first scheduled interview because he experienced car trouble and, upon notifying the researcher, requested to have his entire interview conducted the following day. Through semistructured interview questions, participants added their own opinions and ideas. During the interviews, the researcher conducted member-checking to allow each of the participants the opportunity to clarify their answers and to ensure the participants' answers were being understood correctly by repeating the answers back to them (Miles et al., 2014). 
Table 1. Participant Demographics

\begin{tabular}{cccccc}
\hline Participant & Agency & Position & Gender & Age & Years employed \\
\hline M-1 & A & Supervisor & Female & 28 & 4 \\
M-2 & A & Supervisor & Male & 32 & 3 \\
M-3 & B & Supervisor & Male & 58 & 6 \\
M-4 & B & Supervisor & Male & 27 & 2.5 \\
A & A & DSP & Female & 42 & 4 \\
B & A & DSP & Female & 56 & 10 \\
C & A & DSP & Female & 27 & 5 \\
D & A & DSP & Female & 28 & 3 \\
E & B & DSP & Male & 27 & 3 \\
F & B & DSP & Male & 36 & 2.5 \\
G & B & DSP & Female & 56 & 10 \\
H & B & DSP & Female & 40 & 10 \\
\hline
\end{tabular}

Note. DSP = direct support professional.

\section{Results}

Once the data were analyzed, the themes discovered were grouped into three categories: barriers, culture, and perception. The researcher coded the information from the interview transcripts in relation to the research question and nodes were created in NVivo 12, with categories being matched to the emergent themes. Table 2 reveals these themes, grouped by category, and the number of sources or responders, along with the number of references found. For example, when asking questions on leadership, 11 participants discussed disconnected leadership, which was referenced 33 times.

Table 2. Categories and Themes

\begin{tabular}{lcc}
\hline \multicolumn{1}{c}{ Coded category and its themes } & Sources & References \\
\hline Barriers & & 22 \\
Lack of training & 12 & 15 \\
Personal interpretations & 10 & 21 \\
Lack of policies & 8 & 22 \\
Lack of communication & & \\
Culture & 5 & 11 \\
Evaluations & 12 & 18 \\
Inconsistent implementation & 11 & 33 \\
Disconnected leadership & 11 & 24 \\
Lack of recognition & & \\
Perception & 11 & 26 \\
Low morale & 12 & 20 \\
Low job satisfaction & 8 & 10 \\
High turnovers & & \\
\hline
\end{tabular}

\section{Category 1: Barriers}

Upon analyzing the data, is was evident that there were barriers that led the DSPs from truly grasping and implementing the New York State DSP core competencies. 


\section{Lack of trainings}

There were 22 references made to lack of trainings. There were 11 respondents who had various responses to questions regarding training but ultimately, these 11 participants stated that they did not receive core competencies training, did not remember exactly if they did receive training, or felt training was lacking in some way.

\section{Personal interpretations}

A second barrier that was noted was that all the participants held different interpretations and views of the core competencies and its effectiveness. There were 15 references made to personal interpretations. The participants related that the competencies are implemented as per the staff's own viewpoints and that their supervisors allowed them the free range to implement the competencies as to their own personal values. As such, the competencies were not implemented as prescribed by the standardized training but rather as per the staff's own interpretations.

\section{Lack of policies}

The third theme found was the lack of organizational policies related to the core competencies. There were 21 references made to the lack of policies. Interviews revealed that although the organizations held policies and procedures, none of the participants could remember if or believed that there were any policies or procedures related to the core competencies specifically. In addition, during the interviews, DSPs reported that would like to see written policies regarding the competencies, so they knew exactly what was expected of them.

\section{Lack of communication}

There were 22 references made to issues related to the lack of communication. Supervisors and DSPs both acknowledged issues with communication. Supervisors admitted during interviews that they do not have the time to explain everything to their staff due to the multiple responsibilities they are expected to complete each day. The DSPs, on the other hand, stated that information is not communicated to them from their supervisors, resulting in issues with services and supports. In addition, DSPs also conveyed that conflicting information would often be provided by different supervisors, resulting in confusion in directives. DSPs also added that they believed that their supervisors did not take the time to communicate simply because they did not care about the staff.

\section{Category 2: Culture}

Organizational culture is understood as behaviors shared among members (Glisson \& James, 2002), existing at various levels (Detert et al., 2000) that impacts employees' attitudes and behaviors (Schein, 1996, 2010; Smircich, 1983). Upon reviewing the data, it was clear that the culture of both organizations impacted the way the core competencies were being implemented.

\section{Evaluations}

There were 11 references made to evaluations with one interview being a DSP. All supervisors interviewed stated that the staff performance evaluations were the tool to measure how DSPs were meeting the expectations of implementing the core competencies. The DSPs, however, did not know they were being evaluated on the competencies until they were provided with the form to review and sign. Further, DSPs reported that they felt that their supervisors did not know the core competencies themselves as the competencies were never mentioned throughout the year but only when their evaluations were due.

\section{Inconsistent implementation}

The interviews not only revealed that the core competencies were viewed differently by staff and supervisors, but also that they were implemented inconsistently. There were 18 references made to the inconsistent 
implementation of the core competencies. The DSPs described the inconsistencies of implementing the core competencies as per the competency they knew. For instance, DSPs C, F, and H related they implemented the competencies as to what they believed the person needed or as per the competencies they knew.

\section{Disconnected leadership}

The organizational structure, the function and mindset of an organization's employees is directly related to the core beliefs and actions of the leadership (Chatman \& Cha, 2003). There were 33 references made to disconnected leadership. The data showed that there was a disconnection between the supervisors and the DSPs, which, in turn, impacted the implementation of the core competencies. In addition, the perception of supervisors and DSPs differed. DSPs believed that their supervisors did not engage with them because they did not care about staff. The DSPs either felt that their supervisors did not know enough about their own jobs to help, listen to their concerns or problem solve issues. DSPs also stated that there was a lack of respect from supervisors due to the disparity of roles. For instance, DSP E called himself a "bottom-feeder" to describe his relationship with his supervisor in referring to his role being the lowest position.

Three of supervisors on the other hand, related that they believed they had good leadership skills and stated that they talked and listen to their staff even when they did not have the time. In addition, the supervisors also related that they would work with the staff when needed to allow modeling and assistance. However, Supervisor M-1 believed that even in her position, more layers were needed to promote the core competencies because they were not always available to monitor and provide guidance.

\section{Lack of recognition}

Another theme discovered during the data analysis was the lack of recognition noted by the participants interviewed which impacted their job satisfaction overall. There were 24 references made to lack of recognition. In this instance, DSPs and supervisors in both agencies believed that more recognition was needed. Supervisors believed that DSPs could benefit from recognition awards as to provide incentives and increase motivation. DSPs also felt that their work was not recognized particularly because they deal with client behaviors. DSP A described how she is often made to feel unimportant when dealing with client behaviors, as her supervisor never asks if she is hurt or if she is okay. This DSP believed that her own wellbeing does not matter and simply grew accustomed feeling unappreciated for the difficult work she is sometimes faced with.

\section{Category 3: Perception}

In reviewing the data, it was discovered that the supervisors and DSPs differed in their perceptions of the implementation of the core competencies because of their specific roles. This disconnection in perception impacted morale, job satisfaction, and retention in each of the organizations, thus effecting the implementation of the core competencies.

\section{Low morale}

There were 24 references made to low morale. The supervisors and DSPs all responded as how they believed morale was in their organizations with different views. Supervisors M-1 and M-2 from Agency A both acknowledged that the morale was different and depended if you worked in managerial or if you worked as a DSP, whereas supervisors M-3 and M-4 described morale in continuous fluctuations from high to low and then again to from low to high.

For the DSPs, low morale was associated to a varied of reasons such as DSPs being promoted to supervisory levels and the coworker relationship changing or as the work performance observed did not warrant the 
promotion. Other DSPs stated that low morale was due to lack of organizational policies resulting in inconsistent practices and in DSPs feeling frustrated.

\section{Low job satisfaction}

There were 20 references made to low job satisfaction. When being asked about job satisfaction, Supervisors as well as DSPs described how they enjoyed their jobs but held different views on where improvements could have been made to increase job satisfaction. The data revealed that supervisors and DSPs believed that to improve job satisfaction, improvements of trainings, increased recognition incentives, defined policies and on critical feedback to set forward for job growth were needed.

\section{High turnovers}

There were 10 references made to high turnovers. Supervisors and DSPs described how staff turnovers impacted job satisfaction and morale which also resulted on how the New York State core competencies were being implemented. The Supervisors perceived high turnovers because of regulations changing, changes in management or working relationships. The DSPs on the other hand, perceived high turnovers due to the lack of recognition they felt because of their role.

\section{Documents Reviewed}

Documents were analyzed from both Agencies A and B to explore relationship to themes found during the interviews. Document 1 for Agency A was the organization's residential handbook, which describes the agency's policies, procedures, and expectations. This document mentions the New York State DSP core competencies as one of the topics to be trained but does not specify how often the employee is to obtain the training. This document also revealed the implementation of the employee's evaluation but fails to mention that the evaluations are related to the staff performance as per the New York State core competencies. The second section of this document does provide an outline of the competencies but with no real objectives on how employees are to meet each of the goals of the competencies. There were, however, outlines found on communication tips on how to meet medical regulatory compliance. Document 2 was the organization's overall employee handbook, which described employee benefits, policies, and expectations. This document also identified the core competencies but no mention on how employees were expected to meet the core competencies. Document 3 for Agency A included the participants training transcripts, which were generated through their human resources software, Paycom. A review of the training transcripts revealed that all participants in Agency A were trained in the core competencies except for one. Supervisor M-2's transcript showed he had not received core competencies training in the last 3 years. Document 4 was Agency B's employee handbook, which included policies related to employee benefits and operational procedures but no specified policies on how employees are to adhere to the New York State core competencies. Document 5 entailed the participants' training transcripts, which were generated from their human resources software, Therap. The review revealed that the participants were trained between 2015 and 2016 with two participants showing they were not trained in the competencies. Table 3 illustrates the documents reviewed with a description and Table 4 provides a description of the participants' latest training dates on the core competencies. 
LoPorto, 2020

Table 3. Case Study Documents

\begin{tabular}{ccc}
\hline Agency & Document & Description \\
\hline A & Document 1 & Residential department handbook \\
& Document 2 & Organizations policy and procedure manual \\
B & Document 3 & Training transcripts from Paycom software \\
& Document 4 & Organizations policy and procedure manual \\
& Document 5 & Training transcripts from Therap software \\
\hline
\end{tabular}

Table 4. Participant Training Dates

\begin{tabular}{cccc}
\hline Agency A participants & Training dates & Agency B participants & Training dates \\
\hline M-1 & March 21, 2018 & M-3 & May 23, 2016 \\
M-2 & March 15, 2017 & M-4 & December 18, 2015 \\
A & March 6, 2018 & $\mathrm{E}$ & October 6, 2015 \\
B & April 12, 2018 & $\mathrm{F}$ & October 13, 2015 \\
C & April 18, 2018 & G & No training \\
D & March 14, 2018 & H & No training \\
\hline
\end{tabular}

\section{Discussion}

The purpose of this qualitative case study was to explore the perceptions of DSPs located in New York State regarding the effectiveness of the DSP competencies in their organizations in relationship to job satisfaction and organizational morale. In addition, this study was also aimed to explore what DSPs perceived to be necessary in enhancing the effectiveness of the New York State DSP competencies organizational practices that would improve IDD care and supports. Responses to the research questions were found in the data through categories and themes. Once the data analysis was complete, the data were organized to provide answers to the two research questions.

Regarding the first question ("How do DSPs describe the effectiveness of the New York State DSP competencies in relationship to job satisfaction and organizational morale?"), it is clear that the supervisorDSP relationship impacted the participants' ability to implement the core competencies and also their interpretations of the core competencies themselves combined with the lack of training also impacted how they were implemented. In addition, the perception and experiences between supervisor and DSP effected job satisfaction and morale.

The second interview question ("What do DSPs perceive that it would take to enhance the effectiveness of the New York State DSP competencies organizational practices to improve IDD care and supports?”) described the DSPs and Supervisors own ideas on what they thought was needed to increase the effectiveness and implementation of the core competencies. By analyzing the data, three categories emerged that became apparent to the effective implementation of the core competencies: barriers, culture, and perception. The data revealed similarities and differences between the cases. The relationship between supervisor and DSP is vital to the provision of services and care to the people with IDD living in the community residential homes (Larson \& Hewitt, 2012).

The researcher summarized and interpreted the study's findings using the participants' perceptions and experiences as they related to the research question and to the conceptual framework. Using Donabedian's 
(1980) QOC model for the conceptual framework, three domains are relevant to high-quality client care: structure, process, and outcomes, which were then applied to the to the themes discovered to explore both the organization's structure (barriers), processes (culture), and outcomes (perception) to the implementation of the core competencies.

\section{Quality-of-Care (QOC) Model}

Donabedian's (1980) QOC model identifies structure, processes, and outcomes as the required components for delivering high-quality individualized support care and services. Structure refers to the environment and resources required to provide services, while processes describes the practices used to implement care. The outcomes are the end results actualized by the recipient. Donabedian argued that a good, efficient structure is essential to good processes and good processes are essential to good outcomes. This model was applied to examine the aspects of QOC related to the implementation of the New York State DSP core competencies to the themes discovered that included the organization's structure (barriers), processes (culture), and outcomes (perception).

\section{Structure: Barriers}

By analyzing the data, four themes became apparent related to the organization's structure: lack of trainings, personal interpretations of the core competencies, lack of policies, and lack of communication. These themes posed as barriers for both organizations and their DSPs as there were no real foundations to set forth with the implementation of the core competencies once employees were trained.

\section{Lack of trainings}

Relevant trainings between leaders and employees clarifies expectations and personal interpretations. The data revealed similarities and differences between the cases but showed that continuous trainings may be beneficial for the universal implementation of the competencies. All participants interviewed remembered receiving training on the core competencies when hired, but none of them were able to recall the material or the specific goals of the core competencies.

\section{Personal interpretations}

The participants interviewed all held personal views of the core competencies which also posed as a structural barrier because different interpretations of the competencies meant different forms of implementation. The data revealed that DSPs perceived the core competencies as a basic guideline to assist them in their roles but believed that the core competencies could be applied as they saw fit or to the tools and resources the organization allowed them to have. In addition, interviewees all spoke about being held to the expectation of professionalism, but perceived supervisors being unprofessional themselves which also violated several competencies. Thus, supervisors and DSPs perceived the competencies to be implemented as how they felt should be implemented as there was no governing oversight to ensure its proper implementation.

\section{Lack of policies}

For Agency A, the seven goals of the core competencies were mentioned in the residential manual, but in the scope of what staff was going to be trained on and expected to follow, whereas Agency B held no policies related to the core competencies. The document review revealed that there was no specific reference on how DSPs were to fulfill the expectations of the New York State DSP core competencies. Policies facilitate leaders by providing specific guidelines for their actions and the actions of the employees they are supervising. The findings revealed that there was a lack of policies geared to the implementation of the core competencies. 


\section{Lack of communication}

Participants in the study all revealed issues with communication. Supervisors in the study revealed that they did not always take the time to answer DSPs questions or concerns simply because they did not have the time to do so. The DSPs' on the other hand, noted that supervisors did not take the time to answer their questions or concerns leading them to believe that did not know the answers, did not know how to problem solve a situation or simply that their supervisors did not care about them. Participants also revealed that the lack of communication created confusion among the DSPs effecting how services were provided.

\section{Processes: Culture}

Four themes became apparent related to each of the organization's processes: evaluations, inconsistent implementation of the core competencies, disconnected leadership, and lack of employee recognition. These organizational processes create the culture of the organization. Organizational culture is understood as behaviors shared among members (Glisson \& James, 2002), existing at various levels (Detert et al., 2000), that impact employees' attitudes and behaviors (Schein, 1996, 2010; Smircich, 1983).

\section{Evaluations}

All supervisors interviewed stated that the staff performance evaluations were the tool to measure how DSPs were meeting the expectations of implementing the core competencies. The DSPs, however, were not aware that they were being evaluated on the competencies except for one. One DSP in Agency A was aware that she would be given an evaluation based on the core competencies. However, this DSP has been employed in the agency for 3 years and still has not been given an evaluation. This is one example on how processes are being practiced inconsistently. Supervisor interviews revealed that they were aware that DSPs were not informed that their performance would were based as per the outlined competencies and would only inform them when being given the evaluation.

\section{Inconsistent implementation}

Interviews revealed that the implementation of the core competencies were inconsistently implemented because supervisors and DSPs all viewed and interpreted the core competencies differently. As such, they would apply the competencies as to what specific competency they knew. All participants revealed that there were aware that core competencies held various competencies but only practiced the ones they could apply when a situation required it.

\section{Disconnected leadership}

Leadership directly impacts employee performance and morale. In contrast, ineffective leadership tends to create low organizational commitment and job satisfaction, which impact employee morale (Aboyassin \& Abood, 2013; Gray-Stanley et al. 2010; Gray, \& Muramatsu, 2013). Supervisors and DSPs from both organizations recognized a disconnect in the relationship. Supervisors interviewed recognized that their DSPs wanted to be heard but, due to their own responsibilities and duties, they were not able to dedicate the time to meet with their DSPs. From the DSPs perspective, supervisors simply do not care about them to take the time to meet and talk with them. DSPs also held a perception of themselves as not being worthy enough for supervisors to take time for them or to take them seriously. One DSP from Agency B even referred to himself as a "bottom-feeder," describing himself as being the lowest of the low in the totem pole of the organization's job positions. He also referred his supervisor and administration as the "elite," recognizing that there was a clear, identified line between supervisors and DSPs. This disconnect between supervisors and DSPs also contribute to the lack of communication. 


\section{Lack of recognition}

The disconnect between supervisors and DSPs also allowed for the lack of recognition of DSPs and in the work that they do. All supervisors interviewed revealed that DSPs should receive more praise and recognition but also admitted that it simply does not occur. DSP interviews also revealed their own need to be recognized by their supervisors. Due to the lack of communication and disconnection from their supervisors, DSPs held the perception that their supervisors did recognize them because they did not care or because they themselves did not know or understand the complexities of being a DSP. DSPs interviewed from both agencies believed their supervisors cared more about paperwork than their own safety and well-being due to the lack of recognition when sustaining injuries from working with behavioral individuals.

\section{Outcomes: Perception}

Donabedian's (1980) QOC model identifies structure, processes, and outcomes as the required components for delivering high-quality individualized support care and services. The outcomes in this study related to the supervisors and DSPs own perceptions on how processes and structure impacted the implementation of the core competencies ultimately effecting support care and services.

\section{Low morale}

The concept of morale includes satisfaction with the work environment, enthusiasm, and commitment to the organization (Johnsrud, 1996) while other studies, related morale to employee behaviors (Jackson, Rossi, Rickamer-Hoover \& Johnson, 2012). For this study, morale was defined as "Emotions and attitudes that includes satisfaction with the work environment, commitment or loyalty to the institution and a willingness to work toward common goals" (Johnsrud et al., 2000, p. 47). As such, interviews of supervisors and DSPs revealed that morale was dependent on the position you were in. All four supervisors acknowledged that morale was higher at their level than that of DSPs. One supervisor also noted morale being difficult for DSPs because they never received the respect or recognition they truly deserved. DSPs, on the other hand, noted low morale because of the continuous leadership changes, shifts not being fully staffed and the lack of respect they felt when not having their questions answered or concerns addressed. One DSP even spoke about her feelings in being defeated, for not being able to build a relationship with her supervisor, as she was often ignored nor assisted when needed. Thus, DSPs work their shifts feeling frustrated and devalued. These feelings of being frustrated and devalued mixed with continuous changes and disconnection with leadership, results in inconsistent implementation of the core competencies. Low morale further presented challenges and barriers to the implementation of the core competencies when consequently, the clients receiving support services would be exposed to employee and workplace conflict impacting their care and treatment.

\section{Low job satisfaction}

Job satisfaction is the degree to which job needs are fulfilled and how much of this fulfillment is perceived by the employee (Porter et al., 1974). It is a positive or negative evaluative judgement one makes about one's job or job situation (Weiss, 2002). Job satisfaction is therefore ultimately described as to what an employee feels about his/her job, and how the job itself is perceived. Supervisors and DSPs from both organizations described how they enjoyed their jobs but held different views on where improvements could have been made to increase their job satisfaction. For instance, supervisors wanted to feel less burnt out because they were simply juggling too many responsibilities. They related the feelings of being burnt out to the lack of training DSPS were provided, which meant they had to manage their managerial duties while ensuring shifts were covered. DSPs also believed their job satisfaction would increase by decreasing burn out by working less shifts and being given the opportunity to take vacations when requested. DSPs also believed that their job satisfaction would increase through recognition and promotions. For these DSPs, they wanted their supervisors to recognize, support them and to treat them fairly. One DSP described his interaction with his 
supervisor when requesting time off several times and was denied due to shifts being short staffed but noted, that same supervisor taking three vacations during each time of his requests.

\section{High turnovers}

During the interviews, supervisors and DSPs both described how shifts needed to be covered due to the residences being short staffed. This need for staff was related to low job satisfaction and morale. Supervisors and DSPs from both organizations perceived high turnovers because of low job satisfaction, low morale and ineffective working relationships. Interviews revealed that DSPs did not commit to their jobs because of the lack of recognition, lack of communication and ultimately the inability to build relationships with their supervisors. Supervisors on the other hand, related DSPs turnovers to burnout or finding a better job.

\section{Implications for Social Change}

DSP perceptions on the implementation of the DSP competencies may reveal discrepancies between policy development, implementation, and organizational practices. Sharma and Good (2013) posited that supervisors need to understand the importance of social change in the organization as they play a critical role in setting forth that change. When supervisors focus on social change, they create positive human impact, moral goodness, and unconditional social improvement through and within the organization. However, when there are perceived barriers or policy discrepancies to performance expected, competency implementation and organizational practices becomes misconstrued.

This study explored the process of organizational change because of the New York State DSP competency mandated implementation. Implications for social change may include improving organizational policies and procedures that will align with the guidelines specified in the New York State DSP core competencies. The alignment between organizational policies and the New York State DSP competencies will help improve DSP job satisfaction, work performance, and morale that will ultimately improve IDD services.

\section{Recommendations for Social Change}

The implications of this study have the potential to affect positive social change in several ways. The first contribution that this study makes to positive social change is that this research provides insights into how IDD service provider organizations need to integrate the core competencies into instructional practicum activities. The study identified the need for trainings with a practicum component, so DSPs become knowledgeable and confident in implementing the core competencies. Practicum training with a hands-on approach would allow teaching, learning, and assessment of the core competencies in various everyday, reallife situations that will ultimately benefit the people living in the community residential homes. The second implication for social change is promoting the worth, dignity, and development of DSPs. The results of this study showed that DSPs loved their jobs and enjoyed their positions, but often felt devalued due to the lack of opportunities provided to use their voice for input and due to feelings of being ignored. Employees benefit from improved working conditions in which supervisors show genuine concern that promotes a better sense of work that has meaning and purpose and contributes to the effectiveness of the organization (Hewitt \& Larson, 2007). The last social implication is that this study contributed to the body of knowledge of DSP perception on the implementation of the New York State DSP competencies. The results demonstrated how perception played a role in the implementation of services. DSPs, therefore, need to have a perception of their organization that holds positive human impact, moral goodness, and unconditional social improvement through and within that organization. Improving organizational policies and procedures that aligns with the New York State DSP core competencies, that provides DSP their own platform for organizational inclusion will help improve DSP job satisfaction, work performance and morale. I recommend four potential areas of 
policy adjustments that could benefit from further study: competency and practicum development, communication strategies, shared governance, and employee appreciation strategies.

\section{Competency and Practicum Training Development}

The results of this study showed that training in the core competencies was inconsistent. Some participants did not receive training while, others did not remember if they ever received the training or learned the competencies while on the shift. It is recommended that organizations invest in training programs that allow frequent opportunities for DSPs and supervisors to practice the core competencies. Increased hours of training do not address issues of DSP recruitment, retention, performance, or quality of service. Research shows that competency-based training requires an interactive, hands-on approach that integrates education into daily practice (Stone, 2001; Stone \& Harahan, 2010). Both organizations provided core competency training at new hire training through power point presentations and document reviews but there was no evidence on how implementation of those trainings were followed up on once the staff begun working. As such, both organizations would benefit from core competencies training developed through interactions with peers and supervisors in different learning contexts, such as community residences, day habilitation programs and in the community.

To support DSPs in learning the core competencies and then in implementing them, trainers must provide authentic and frequent opportunities to practice these skills, which include role plays, collaborative work, conflict resolution, and problem-solving opportunities. As such, core competencies trainings at new hire also needs to be supplemented with core competencies training in the field, while on the job. Mentoring and teaching techniques can be added in developing the staff on how, when and where to apply the core competencies as so the DSP can fully grasp what their role is. Thus, with a practicum component, the staff can learn how to appropriately apply the core competencies to real-life situations.

\section{Communication Strategies}

The results of this study suggest that communication is vital between supervisors and DSPs to ensure adequate care and support for the individuals being served but, also to build effective relationships. The study showed that miscommunication led to confusion in DSP responsibilities and the perception that supervisors simply did not care about them. This perception created a disconnect between the supervisors and DSPs. In addition, this failed communications between DSPs and their supervisors were also compounded by the negative experiences DSPs had with their supervisors. Organizations need to develop communication policies that outline the specific actions supervisors will take in addressing DSP concerns. At the same time, these communication policies need to provide strategies that allows for DSP and supervisor supportive dialogue that may enhance DSP performance such as through consistent evaluations. In addition, it will also help minimize misinterpretations of services addressing the theme of personal interpretations. The results of study showed that because the core competencies were not fully or consistently communicated, DSPs implemented the competencies as per their own interpretations. Supervisors need to set time aside for their DSPs and policies need to be created to promote and influence that time. DSPs vary in their levels of need for support and their ability to connect with their supervisor will be dependent on passed interactions. By building communication strategies within a supportive dialogue however, effective communication can be built, setting forth for effective IDD services but also for increased job satisfaction, morale, and retention.

\section{Supervisor/DSP Collaborative Forums}

A recurring theme found in this study was that DSPs felt disconnected from their supervisors. As already recommended, open and clear lines of communication are needed to allow for consistent implementation of 
the core competencies but also, for the building of working relationships. Supervisors and DSPs, however, must also be given the opportunity to engage in informal, transparent, and interpersonal collaborative forums. The findings of this study showed that the DSPs believed that their supervisors perceived them as unimportant because they were never given a platform to discuss their own concerns, issues, or goals. Willard-Grace et al. (2014) found organizations that fostered collaborative-team culture experienced less burn-out and exhaustion than teams who did not foster close, collaborative teams. In addition, having collaborative dialogue between supervisors and DSPs allowed for supportive, valued, and engaged employees (Hewitt \& Larson, 2007). Thus, as DSP needs and expectations are fulfilled, job satisfaction increases. As such, it is recommended that strategies for supervisor/DSP forums be developed.

\section{Employee Recognition Strategies}

Another recurring theme found in this study was the lack of recognition or appreciation the DSPs perceived from their employers. Organizational leaders need to develop employee incentive programs that recognize their employees, which may increase employee morale. The results of this study showed that DSPs may not have fully engaged in the implementation of the core competencies because they perceived their work to be unrecognized and unappreciated. In turn, morale and job satisfaction were low because DSPs believed that they were not valued. Participants identified leadership as needing to be more responsible in creating personal recognition programs for work well noted rather than annual recognition programs where the recognition were more generic. These incentives can range from small tokens of appreciation to large recognition platforms, but the key is to recognize the staff and to make it personal. Supervisors need to become creative in recognizing their staff as soon as recognition should be noted. There are many reasons behind low staff morale, but the primary reason discovered was poor and ineffective job recognition and leadership. Thus, this poor and ineffective leadership led to DSPs low morale, low job satisfaction which also led to DSP lack of engagement and commitment to the implementation of the core competencies.

\section{Conclusion}

This study addressed two research questions. The first surrounded the DSPs description on the effectiveness of the New York State DSP core competencies in relationship to their job satisfaction and morale. The cases demonstrated how organizational barriers such as lack of trainings, communication and policies influence job satisfaction and morale which impacts the implementation of the core competencies. The second research questions explored DSP perception on what was needed to enhance New York State DSP competencies organizational practices to improve IDD care and supports. Supervisors and DSPs believed that the disconnect and lack of DSP recognition played a role in organizational practices. To enhance the core competencies, supervisors and DSPs need to work in collaborative-team platforms with streamlined policies and procedures that includes a practicum component to trainings, effective communication and appreciation strategies.

Implications for future research may also include supervisor and DSP pay scale analysis, how shared governance impacts the organization, formal workload comparison between DSP and supervisors, and a formal analysis on organizational financial loss as a result of DSP turnovers.

As people with IDD are now part of our communities, organizations must successfully train DSPs with the skills, knowledge, and confidence to implement the New York State DSP core competencies for everyday life. To do so, organizations must provide an environment free of contradictory policies but, develop policies that embraces open communication, collaboration and recognition within all job levels that will only optimize the care and services being provided to the people living with IDD. 


\section{References}

Aboyassin, N. A., \& Abood, N. (2013). The effect of ineffective leadership on individual and organizational performance in Jordanian institutions. Competitiveness Review: An International Business Journal, 23(1), 68-84

Avolio, B. J., Walumbwa, F. O., \& Weber, T. J. (2009). Leadership: Current theories, research, and future directions. Annual Review of Psychology, 6o, 421-449. https://doi.org/10.1146/annurev.psych.60.110707.163621

Berson, Y., \& Linton, J. D. (2005). An examination of the relationships between leadership style,quality, and employee satisfaction in R\&D versus administrative environments. R\&D Management, 35(1), 51-60.

Burchard, S. N., Pine, J., \& Gordon, L. R. (1990). Manager competence, program normalization and client satisfaction in group homes. Education and Training in Mental Retardation, (32)2, 277-285.

Cameron, K. (2011). Responsible leadership as virtuous leadership. Journal of Business Ethics, 98(1), $25-35$.

Camilleri, D., \& O'Callaghan, M. (1998). Comparing public and private hospital care servicequality. International Journal of Health Care Quality Assurance, 11(4), 127-133.

Chatman, J. A., \& Cha, S. E. (2003). Leading by leveraging culture. California management review, 45(4), 20-34.

Chun, J. U., Sosik, J. J., \& Yun, N. Y. (2012). A longitudinal study of mentor and protégé outcomes in formal mentoring relationships. Journal of Organizational Behavior, 33(8), 1071-1094.

Creswell, J. (2009). Research design: Qualitative, quantitative, and mixed methods approaches. Sage.

Detert, J. R., Schroeder, R. G., \& Mauriel, J. J. (2000). A framework for linking culture and improvement initiatives in organizations. Academy of Management Review, 25(4), 850-863.

Donabedian, A. (1980). The definition of quality and approaches to its assessment. Health Administration Press

Glisson, C., \& James, L. R. (2002). The cross-level effects of culture and climate in human service teams. Journal of Organizational Behavior, 23(6), 767-794. https://doi.org/10.1002/job.162

Godshalk, V. M., \& Sosik, J. J. (2000). Leadership styles, mentoring functions received, and job-related stress: A conceptual model and preliminary study. Journal of Organizational Behavior, 21(4), 365-390.

Gosling, J., \& Mintzberg, H. (2003). The five minds of a manager. Harvard Business Review, 81(11), 54-63.

Gray, J. A., \& Muramatsu, N. (2013). When the job has lost its appeal: Intentions to quit among direct care workers. Journal of Intellectual and Developmental Disability, 38(2), 124-133.

Gray-Stanley, J. A., Muramatsu, N., Heller, T., Hughes, S., Johnson, T. P., \& Ramirez-Valles, J. (2010). Work stress and depression among direct support professionals: The role of work support and locus of control. Journal of Intellectual Disability Research, 54(8), 749-761.

Hewitt, A. (1998). Community residential core competencies: Necessary competencies for direct support staff working in community residential services for people with developmental disabilities. University of Minnesota

Hewitt, A., \& Lakin, K. C. (2001). Issues in the direct support workforce and their connections to the growth, sustainability and quality of community supports: A Technical Assistance Paper of the National Project: Self-Determination for People with Developmental Disabilities. University of Minnesota. 
Hewitt, A., \& Larson, S. (2007). The direct support workforce in community supports to individuals with developmental disabilities: Issues, implications, and promising practices. Mental Retardation and Developmental Disabilities Research Reviews, 13, 178-187. https://doi.org/10.1002/mrdd.20151

Hewitt, A. S., Larson, S. A., Lakin, K. C., Sauer, J., O'Nell, S., \& Sedlezky, L. 196 (2004). Role and essential competencies of the frontline supervisors of direct support professionals in community services. Mental Retardation, 42(2), 122-135

Jackson, E. M., Rossi, M. E., Rickamer Hoover, E., \& Johnson, R. E. (2012). Relationships of leader reward behavior with employee behavior: Fairness and morale as key mediators. Leadership \& Organization Development Journal, 33(7), 646-661.

Jabnoun, N., \& Chaker, M. (2003). Comparing the quality of private and public hospitals. Managing Service Quality, 13(4), 290-299.

Johnsrud, L. K. (1996). Maintaining morale: A guide to assessing the morale of midlevel administrators and faculty. College and University Personnel Association.

Johnsrud, L. K., Heck, R. H., \& Rosser, V. J. (2000). Morale matters: Midlevel administrators and their intent to leave. The Journal of Higher Education, 71(1), 34-59.

Konrad, T. R., \& Morgan, J. C. (2006). Step up now for better jobs and better care: The evaluation of a workforce intervention for direct care workers. University of North Carolina. http://www.shepscenter.unc.edu/wpcontent/uploads/2013/07/040406 UNC Ex Summary Final trk.pdf

Larson, S.A., \& Hewitt, A. (2012). Staff recruitment, retention and training strategies for human community service organizations. Institute of Community Integration, University of Minnesota. https://ici.umn.edu/products/docs/Staff Recruitment book/Staff Recruitment book.pdf

Larson, S. A., Hewitt, A. S., \& Knobloch, B. (2005). Recruitment, retention and training challenges in community human services. University of Minnesota. https://ici.umn.edu/products/docs/Staff_Recruitment_book/Staff_Recruitment_book.pdf

Lawthers, A. G., Pransky, G. S., Peterson, L. E., \& Himmelstein, J. H. (2003). Rethinking quality in the context of persons with disability. International Journal for Quality in Health Care, 15(4), 287-299.

Lohr, K. N., \& Schroeder, S. A. (1990). A strategy for quality assurance in Medicare. New England Journal of Medicine, 322(10), 707-712.

Maynard, M. T., Gilson, L. L., \& Mathieu, J. E. (2012). Empowerment-fad or fab? A multilevel review of the past two decades of research. Journal of Management, 38(4), 1231-1281.

McConkey, R., \& Collins, S. (2010). The role of support staff in promoting the social inclusion of persons with an intellectual disability. Journal of Intellectual Disability Research, 54(8), 691-700.

McDermott, A., Kidney, R., \& Flood, P. (2011). Understanding leader development: learning from leaders. Leadership \& Organization Development Journal, 32(4), 358-378.

Miles, M. B., Huberman, A. M., \& Saldaña, J. (2014). Qualitative data analysis: A methods sourcebook. Sage Publications.

National Alliance for Direct Support Professionals. (2011). 15 Areas of competencies: The NADSP code of ethics. https://www.nadsp.org/dsp-credentialing/15-competency-areas/10-library.html

Office for People With Developmental Disabilities. (2013). The road to reform: Putting people first. http://www.lwarc.org/OPWDD Road to Reform April2013.pdf 
Paraprofessional Health Institute. (2013). State by state demand for direct care workers, 2010-202O. http://phinational.org/fact-sheets/state-state-projected-demand-edition.new-direct-care-workers$\underline{2010-20}$

Patton, M. Q. (2002). Qualitative research and evaluation methods (3rd ed.). Sage.

Porter, L. W., Steers, R. M., Mowday, R. T., \& Boulian, P. V. (1974). Organizational commitment, job satisfaction, and turnover among psychiatric technicians. Journal of Applied Psychology, 59(5), 603-609.

Ranji, S. R., Shetty, K., Posley, K. A., Lewis, R., Sundaram, V., Galvin, C. M., \& Winston, L. G. (2007). Closing the quality gap: A critical analysis of quality improvement strategies, Vol. 6: Prevention of healthcare-associated infections. Agency for Healthcare Research and Quality.

Rizzolo, M. C., Friedman, C., Lulinski-Norris, A., \& Braddock, D. (2013). Home and community based services waivers: A nationwide study of the states. Intellectual and Developmental Disabilities, 51(1), 1-21.

Rothman, D. J., \& Rothman, S. M. (1984). The Willowbrook wars: A decade of struggle for social justice. Harper \& Row.

Schein, E. H. (1996). Culture: The missing concept in organization studies. Administrative Science Quarterly, $41(2), 229-240$.

Schein, E. H. (2010). Organizational culture and leadership (4th ed.). Wiley.

Sharma, G., \& Good, D. (2013). The work of middle managers: Sense-making and sense-giving for creating positive social change. The Journal of Applied Behavioral Science, 49(1), 95-122.

Smircich, L. (1983). Concepts of culture and organizational analysis. Administrative Science Quarterly, 28(3), 339-358.

Stake, R. E. (1995). The art of case study research. Sage.

Stone, R. I. (2001). Who will care for us? Addressing the long-term care workforce crisis: The Urban Institute. http://www.urban.org/sites/default/files/publication/42696/310304-Who-Will-Care-ForUs-Addressing-the-Long-Term-Care-Workforce-Crisis.PDF

Stone, R., \& Harahan, M. F. (2010). Improving the long-term care workforce serving older adults. Health Affairs, 29(1), 109-115.

Thousand, J. S., Burchard, S. N., \& Hasazi, J. E. (1986). Field-based generation and social validation managers and staff competencies for small community residences. Applied Research in Mental Retardation, 7(3), 263-283.

Tuckey, M. R., Bakker, A. B., \& Dollard, M. F. (2012). Empowering leaders optimize working conditions for engagement: A multilevel study. Journal of occupational health psychology, 17(1), 15-27.

Valla, R. (2014). Service provider implementation of the national alliance for direct support professionals (NADSP) Code of Ethics, NYS DSP Core Competencies and NYS DSP Performance Evaluations [Memorandum]. Office for People With Developmental Disabilities.

Weiss, H. M. (2002). Deconstructing job satisfaction: Separating evaluations, beliefs and affective experiences. Human Resource Management Review, 12(2), 173-194.

Willard-Grace, R., Hessler, D., Rogers, E., Dubé, K., Bodenheimer, T., \& Grumbach, K. (2014). Team structure and culture are associated with lower burnout in primary care. The Journal of the American Board of Family Medicine, 27(2), 229-238.

Yin, R. K. (2014). Case study research: Design and methods (5th ed.). Sage. 
LoPorto, 2020

\section{Appendix}

\section{New York State Direct Support Professional Core Competencies}

\section{Goal 1: Putting People First}

Competency Area A: Support a person's unique capacities, personalities, and potential Skills:

1. Demonstrates respect for all individuals being supported

2. Demonstrates support for individual choice-making in order to enhance confidence and assertiveness

Tasks:

- Communicates directly with individuals

- Uses person-first language when communicating with the individual.

- Uses body language and eye-contact to show attention to others.

- Monitors own tone of voice and volume when providing instruction and direction to individuals.

- Assists individuals to dress and groom in a way that demonstrates his/her self-respect and dignity.

- Consistently uses person-first language when communicating about the individual.

- Develops a respectful and genuine relationship with the individual that is demonstrated through tone of voice, interpersonal interactions, and content of conversations.

- Supports choices made by the individual while considering health and safety concerns.

- Demonstrates the use of positive feedback

- Recognizes and supports choices made by the individual while considering health and safety concerns

- Provides positive feedback and encouragement to the person supported as the person assumes his/her leadership role in choice-making

- Assists individuals in sorting through choices

\section{Competency Area B: Getting to know the person through assessment and discovery} Skills:

1. Evaluates the ways in which past and current events, and environmental factors, affect the way the person acts/reacts to others.

2. Using a holistic approach, participates in the individual's life planning activities, and assists in their implementation.

3. Encourages and supports problem-solving and coping skills.

4. Is informed about formal and informal assessment and can conduct informal assessments in a variety of settings, to gain information about the individual and his/her response to the environment.

5. Supports the self-direction of services. Tasks:

- Reviews files and relevant information.

- Meets with the individual and their circle of support to learn more about the person. 
- Recognizes that challenging behaviors can be a form of communication and responds to it appropriately.

- Implements goals as written to achieve desired outcomes.

- Has access to and can interpret and question the plans.

- Is able to respectfully contribute, within the team setting, to the identification of desired plans for an individual.

- Talks about problems/concerns with the individual to gain understanding of his/her point of view

- Helps the person better cope with their problem by providing emotional support.

- Can demonstrate the use of informal assessment techniques used on a daily basis in each setting in which he/she works with individuals (home, work, travel, neighborhood, etc.), such as observation and active listening.

- Can describe the concept of self-determination and how it applies to the person receiving support.

\section{Competency Area C: Promoting advocacy with the individual}

Skills:

1. Seeks information on the range of services available to individuals with developmental

2. disabilities.

3. Provides opportunities for the individual to be a self-advocate.

4. Performs advocate responsibilities while demonstrating respect for the processes and people involved.

5. Describes and supports individuals' rights and responsibilities.

6. Identifies when an individual's rights may have been breached and takes action to prevent, stop and report the possible breach.

Tasks:

- Is able to describe, in general terms, categories of services available.

- Can describe the basic structure of the services available for people with developmental

- disabilities to meet the individual's needs and desires, and is able to advocate for

- additional services, as needs arise.

- Clearly communicates suggestions to team members for types of services and supports that an individual need and/or wants.

- Can state who to contact to find out about various services from which the person can benefit.

- Encourages and assists the individual to express on his/her own behalf.

- Is able to describe the individual's rights to due process through the agency's human rights committee.

- Can identify who to contact when advocacy questions arise.

- Follows the appropriate communication and supervisory channels when initiating change or change recommendations.

- Is able to discuss the rights and responsibilities to which any individual is entitled.

- Can discuss the challenges faced by individuals with developmental disabilities regarding their rights. 


\section{Competency Area D: Facilitating personal growth and development}

Skills:

1. Demonstrates the ability to effectively teach skills to people supported.

2. Recognizes the individual's need for teaching, and preferred style for learning, and can perform individualized teaching based on this information.

3. Assesses the effectiveness of formal and informal teaching provided and makes adaptations where needed.

Tasks:

- Demonstrates the ability to follow a plan for successful teaching.

- Takes advantage of informal opportunities to teach.

- Is able to teach in a group setting.

- Is able to identify the effectiveness of the teaching plans.

- Can describe the way in which the individual prefers to learn.

- Listens to and observes the individual, while he/she performs skills related to teaching provided, to determine if the individual has learned the desired skill.

\section{Competency Area E: Facilitation of supports and services}

Skills:

1. Assists in the development, implementation and on-going evaluation of service plans that are based on the individual's preferences, needs and interests.

2. Continuously shares observations, insights, and recommendations with the individual and his/her support team.

Tasks:

- Is able to implement service plans, as written.

- Continuously evaluates the service plans and makes recommendations, as needed.

- Engages the individual in service planning discussions and activities.

- Shares information in an organized, timely and sensitive manner.

- Shares direct input from the individual and his/her support team members.

\section{Goal 2: Building and Maintaining Positive Relationships}

\section{Competency Area F: Building and maintaining relationships}

Skills:

1. Supports individuals to overcome barriers and challenges to establishing and maintaining a network of relationships and valued social roles.

2. Demonstrates the ability to identify the individual's personal strengths, interests and needed supports for community involvement.

3. Demonstrates strategies to encourage and build the individual's self-confidence. 
LoPorto, 2020

Tasks:

- Encourages the use of social skills to develop and maintain positive relationships.

- Follows the sexual consent status and values of the individual being supported.

- Assists in teaching social skills to develop and maintain positive relationships.

- Supports the person in exploring and practicing faith, religion, spiritual and cultural interests without personal bias.

- Identifies likes and dislikes and matches interests and people with available events and activities in the neighborhood and community.

- If the person desires, supports the person to choose a method to observe his/her faith/religion/spirituality/culture/ethnicity, and make connections with other community members without staff imposing their own values.

- Based upon the individual's desires, supports the person to become a valued member and active participant in groups in his/her faith/spiritual community by looking for opportunities for the person to be included in spiritual activities with their ethnic/cultural group.

- Assists the individual to recognize and take pride in his/her abilities and achievements.

\section{Competency Area G: Creating meaningful relationships}

Skills:

1. Uses a range of effective communication strategies and skills to establish a collaborative relationship with the person.

2. DSP modifies own communication to ensure understanding and respect.

3. Develops trust by communicating empathetically.

4. Recognizes the impact of the possible discrepancies between the individual's chronological age and developmental age when communicating.

Tasks:

- Uses a polite tone of voice.

- Encourages the person to express him/herself.

- Recognizes and respects individual's need for periods of quiet, non-communication time.

- Speaks, models, signs, shows pictures and objects or uses adaptive equipment in ways. that the person understands, according to their plan

- Identifies likes and dislikes, wants and needs, by the person's verbal and non-verbal

- communication as well as in context with personal history and input from friends, relatives and professionals.

- Includes the individual in the conversation, by speaking with the individual, not about the individual.

- Avoids making assumptions about an individual's cognitive abilities based on his/her communication abilities.

- Uses a variety of communication techniques to meet the individual's needs.

- Demonstrates caring through body language, tone, and providing adequate time for communication.

- Demonstrates active listening by repeating words or gestures, asking questions, and validating feelings. 
LoPorto, 2020

\section{Goal 3: Demonstrating Professionalism}

\section{Competency Area H: Developing professional relationships}

Skills:

1. Demonstrates respect in all professional relationships.

Tasks:

- Respects friends and family members through his/her actions and words.

- Actively listens to and take actions related to expressed concerns and passes information along to appropriate personnel members.

- Demonstrates tolerance and acceptance with others

- Develops positive and productive relationships with his/her coworkers, supervisor, and other colleagues.

- Is able to empathize and effectively communicate with family and friends of the individual.

\section{Competency Area I: Exhibiting professional behavior}

Skills:

1. Demonstrates the following desirable professional qualities in the worksite: professional demeanor, attention to punctuality and attendance policies, reliability, flexibility, and pleasantness.

Tasks:

- Demonstrates courtesy to others and contributes to a positive team atmosphere.

- Complies with agency regulations and policies related to dress, confidentiality, professional appearance and use of electronic devices.

- Arrives at work on time, limits use of unscheduled absences, accurately signs in and out.

- Continuously engages in productive activity while at work.

- Is open to doing things in a variety of ways.

- Serves as a positive role model and team member.

- Respects personal and professional boundaries.

- Follows through on all projects and responsibilities.

- Readily adapts to changes in work assignments.

- Approaches problems in a solution-oriented manner.

- Diverts communication related to problems and dissatisfaction from peers to appropriate. channels to effect improvement or resolution.

\section{Competency Area J: Showing respect for diversity and inclusion}

Skills:

1. Demonstrates respect in all matters relating to diversity and inclusion

2. Demonstrates the awareness, attitude, knowledge and skills (i.e. cultural competence) required to provide effective support to those we serve from any ethnic, racial, sexual orientation, religion, gender, socio-economic, age or disability group, as well as any other component diversity groups.

Tasks:

- Shows respect for others' values without imposing their own

- Demonstrates a willingness to accept and respect all components of human diversity 
- Treats individuals served, families and co-workers equitably

- Can articulate personal biases and does not let their personal biases affect their work and seeks support when needed

- Can describe cultural biases and personal differences that might have an effect on interpersonal relationships when working with individuals, families and co-workers/team members.

- Demonstrates the cultural competence required to provide effective support to those we serve.

- Can discuss the concepts of fairness and respect, and the impact that discrimination based on disability, race, gender, religion, etc., has on people.

- When the DSP recognizes that an individual is being discriminated against, he/she is able to serve as an ally to the individual by intervening to stop the inappropriate comments/actions against the individual.

- When a DSP recognizes that an individual is being discriminated against, he/she reports it according to agency procedure.

- Can effectively communicate with those we support regarding their abilities and challenges they may face.

- Demonstrates sensitivity to the lasting effects that discrimination can have on individuals.

- Supports culture and gender specific preferences for health and personal care in accordance with agency policy.

- Identifies and reports the possible disparities in health care delivery that often negatively impact the individuals supported.

\section{Competency Area K: Creating meaningful documentation records}

Skills:

1. Maintains accurate records by collecting, compiling, evaluating data and submitting it in a timely manner to the appropriate sources.

Tasks:

- Notes are recorded in the proper place and in the proper format.

- Notes are signed and dated, according to agency policy.

- Documentation is thorough, including data where required, baseline information, etc.

- Documentation is done on time, according to agency policy.

- Maintains standards of confidentiality and ethical practice.

- Recorded communication should reflect progress and choices made in a manner that would be clearly understood by a reader unfamiliar with the person or program.

- Clearly and effectively communicates information through his/her documentation practices.

\section{Competency Area L: Education, training, and self-development activities}

Skills:

1. Demonstrates enthusiasm for learning the knowledge and skills required to perform the job.

2. Readily seeks and accepts feedback to improve performance.

3. Applies knowledge and skills gained to the job.

Tasks:

- Attends, actively participates in, and successfully completes all required training sessions

- Asks mentors and supervisors to share best practices.

- Is open to and accepting of developmental feedback. 
LoPorto, 2020

- Seeks to learn from mistakes; avoids defending mistakes.

- Discusses application of skills with supervisor/mentor prior to use.

- Demonstrates the ability to learn and apply new and innovative techniques.

- Demonstrates the skill to his/her designated experienced staff or supervisor.

- Receives feedback and applies it to improve skill proficiency on the job.

\section{Competency Area M: Organizational participation}

Skills:

1. Adheres to and promotes the mission, culture and practices of the organization

2. Participates in the work of the organization in a positive way by using problem solving skills.

3. Adheres to corporate compliance policies and procedures.

Tasks:

- Is able to articulate the agency mission and culture in his/her own words and describe how his/her job and everyday activities help support the agency mission.

- Is able to apply, demonstrate, and incorporate the agency mission and culture into everyday practice.

- Participates in the identification of problems.

- Participates in the identification of the causes of problems.

- Actively participates in the identification of solutions.

- Examines options and is open to input.

- Successfully completes training on corporate compliance topics.

- Can access the organization's corporate compliance procedures documents.

- Follows the organization's corporate compliance procedures.

- Recognizes and reports fraudulent behaviors.

\section{Competency Area N: Exhibiting ethical behavior on the job}

Skills:

1. Knows, understands and follows the NADSP Code of Ethics.

Tasks:

- Can access and discuss the 9 aspects NADSP Code of Ethics:

- Primary allegiance is to the person receiving support

- Supports the physical, emotional and personal well-being of the person receiving services

- Shows integrity and responsibility by assisting people to live self-directed lives while, fostering a sense of partnership with the person supported

- Respects and safeguards the confidentiality and privacy of the people served

- Promotes and practices justice, fairness and equity for people served while affirming human and civil rights and responsibilities

- Shows respect for the uniqueness of each person served and value for the persons unique qualities

- Assists people served to develop and maintain meaningful relationships with other people

- Support the persons served to direct the course of their own lives

- Advocates for the people supported for justice, inclusion and full community participation

- Seeks out clarification when not sure about issues around ethics.

- Begins to put the NADSP Code of Ethics into practice.

- $\quad$ Routinely puts the NADSP Code of Ethics into practice. 


\section{Goal 4: Supporting Good Health}

\section{Competency Area O: Promoting positive behavior and supports}

Skills:

1. Demonstrates teamwork with the individual, co-workers and family in implementing positive behavioral support strategies consistent with available behavior support plans.

2. Demonstrates effective methods to teach positive behaviors and support existing positive behaviors.

3. Assess strategies to evaluate how environmental factors affect behavior.

Tasks:

- Accepts and uses feedback to implement positive behavior supports.

- Provides feedback on the effects of the approaches taken.

- Encourages and recognizes positive behaviors by using praise and various reinforcers effectively.

- Is a role model for positive behavior.

- Uses the preferred mode of communication to offer cues to promote positive behaviors.

- Can articulate ways in which environmental factors can have an impact on behavior.

- Proactively reduces previously identified stressful environmental factors such as noise, light, and heat.

\section{Competency Area P: Supporting health and wellness}

Skills:

1. Demonstrates and assists in nutritious meal planning and food preparation, storage and handling procedures.

2. Demonstrates knowledge and understanding of an individual's medical, physical, psychological, and dental health care needs.

3. Demonstrates knowledge of and uses accepted methods to prevent illness and disease and teaches prevention methods to the individual.

4. Recognizes and responds in a timely manner to signs and symptoms of illness/injury and medical emergencies.

5. Provides a safe and clean environment for the individual based on skill level and risks.

6. Accurately documents and adequately protects all health information.

7. Understands and can implement daily health practices to support good health.

Tasks:

- Teaches dining skills according to the individual's needs.

- Assists individuals to use clean, healthy practices when preparing meals

- Adheres to allergy alerts, texture, portion size, and other alerts related to the special requirements of the individual.

- Can discuss the health care information needed to support that person.

- Reviews the person's plan of nursing services to gain a better understanding of the individual's health care needs.

- Can describe general changes in behavior that could be a sign of a possible health-related concern.

- Assists and advocates for individual, as needed and appropriate, to facilitate and optimize informed health care services.

- Assists individual in the safe use and maintenance of adaptive equipment. 
- Follows and can articulate the reasons for procedures that support special populations; such as aging individuals, individuals with diabetes, Prader-Willi syndrome, Autism Spectrum disorders, and those with dual diagnoses.

- Able to understand person's normal behavior and recognizes changes that may indicate health concerns.

- Communicates observed health care concerns to the necessary support network.

- Can state why a person is receiving a specific medication or treatment, as well as the intended effects of that medication or treatment.

- Monitors and reports any adverse side effects of medication or treatments provided.

- Assists, as needed, in healthcare activities of daily living (ADLs), such as oral hygiene and personal care.

- Successfully achieves Medication Administration Certification (AMAP), if required by the individual, support setting or agency policy.

- If Medication Administration Certified (AMAP), the DSP assures that medications are accurately administered and recorded in keeping with agency policy and professional performance standards.

- Can discuss ways in which healthy personal care and hygiene practices prevent illness.

- Is able to identify when an individual is experiencing an illness or injury and responds according to established protocols.

- Able to access emergency phone numbers, such as 911 or EMS.

- Achieves and maintains CPR, first aid and other certifications according to agency policy.

- Assists in securing needed medical appointments in a timely manner (scheduling, arranging transportation, supporting questions and explanations, following agency protocols on consult sheets, documentation, etc.)

- Correctly uses standard precautions, especially hand washing, and can explain the underlying concepts of personal and environmental contamination.

- Uses personal protective equipment (PPE), such as gloves, gowns and masks, when appropriate.

- Frequently cleans and requests replacement of toothbrushes, vaporizers/humidifiers and other ordinary and specialty equipment according to the individual's health plan, standard medical practice, and the manufacturer's instructions.

- Documents the individual's health status, medications, medical needs and appointments, as required.

- Maintains and protects all protected health information (PHI) as directed by the HIPAA legislation.

- Uses appropriate and safe turning, positioning and transfer techniques to support skin and bone integrity and effectively meet individual's unique needs.

- Demonstrates holistic approaches that recognize importance of practices as it relates to appropriate and adequate diet and nutrition, rest and exercise, stress reduction, and smoking cessation.

- Correctly completes routine and/or urgent health care practices such as tube feeding, insulin administration, colostomy and/or catheter care, and Epi-pen administration.

\section{Competency Area Q: Preventing, recognizing, and reporting abuse}

Skills:

1. Recognizes concepts related to the prevention of abuse.

2. Is able to prevent abuse.

3. Correctly follows procedures for mandated reporting and responding.

Tasks:

- Can identify abuse as described in the regulations.

- Can discuss the possible impact of abuse on the person.

- Can prevent, stop, safeguard against, and report abuse according to the OPWDD policy. 
- Develops a deeper understanding of an individual and can describe how changes in his/her mood, interpersonal interactions, and behavior could be an indicator of abuse.

- Can provide examples of the range and nuances of abuse and respond according to agency and OPWDD policy.

- Can effectively intervene so that abuse does not occur

- Can identify triggers and warning signs that indicate abuse might be likely to occur.

- Assists the team and individual to put in place a plan to prevent further incidences.

- Fulfills their obligation to report possible abuse regardless of who allegedly committed the abuse.

- Reports possible abuse to the appropriate person in a timely manner.

- Cooperates with the investigative process.

\section{Goal 5: Supporting Safety}

\section{Competency Area R: Supporting safety}

Skills:

1. Demonstrates skill in applying the principles and practices of the OPWDD PROMOTE (Positive Relationships Offer More Opportunities to Everyone)

2. Demonstrates respect for the safety of all others.

Tasks:

- Supports the individual's connections to others, self-confidence and opportunities for relaxation and recreation (Green Zone) to decrease the possibility of a crisis occurring.

- When the individual is unable to cope with stress (Yellow Zone), the DSP is able to effectively use the following R-Star techniques: Reassessment, Reassurance, Repeat-Ask-Validate, Remind, and Restore.

- Can discuss an individual's vulnerabilities, strengths and potential irritants and effective supports.

- Intervenes effectively when a person is a danger to him/herself and/or others (Red Zone).

- Works to repair and restore the environment and peoples' emotions after a crisis situation (Red Zone).

- Intervenes in a crisis by managing the physical and social environment in an attempt to de-escalate the situation and promote the safety of the individual, co-workers and others.

- Participates in the review of crisis situations with the individual, families and team members to determine the need for ongoing supports and make plans to avoid future crises.

\section{Competency Area S: Supporting safety}

Skills:

1. Supports the safety of all individuals in everyday situations.

2. Follows proper safety procedures in transportation situations.

Tasks:

- Is able to operate emergency equipment, as required.

- Reports to appropriate personnel any detected problem with emergency equipment, or the need for emergency supplies.

- Seeks out and reports potential hazards related to fire, ice, etc.

- Adheres to agency policies, requirements and regulations.

- Can properly operate transportation equipment, such as the lift, and secure wheelchairs, oxygen, and other equipment.

- If operating a vehicle, maintains a current NYS driver's license consistent with agency requirements. 
- Operates the vehicle in a safe and courteous manner consistent with New York State driving laws.

\section{Competency Area T: Ensuring safety of individuals during environmental emergencies}

Skills:

1. Understands and can carry out plans for responding to environmental emergencies.

Tasks:

- Can describe and implement the personal protection plan based on the needs of the individual being supported.

- Is aware of and can execute specific emergency preparedness plans for the location in which he/she works.

- Actively participates in and documents the fire escape drills conducted in the location, according to agency policy.

\section{Goal 6: Having a Home}

\section{Competency Area U: Supporting people to live in the home of their choice}

Skills:

1. Supports the individual by supporting a comfortable home environment.

2. Supports daily activities and accesses additional skilled supports as needed.

Tasks:

- Demonstrates respect by acknowledging that the location is the individual's home or the individual's family home, not the staff's work site.

- Can describe the physical environment of the support setting.

- Follows the rules and guidelines in the home.

- Can describe the individual's daily routine and assists with the routine based on the individual's needs and desires.

- Assists the individual with routine household chores according to the individual's needs (i.e. changing light bulbs, placing decorations outside, etc.).

- Assists the individual to develop his/her skills and activities based on the abilities and

- needs of the individual.

- Assists the individual to become as self-sufficient as possible with transportation needs and refers for travel training when necessary.

- Assists the individual to develop his/her household management skills, based on the individual's needs.

\section{Goal 7: Being Active and Productive in Society}

\section{Competency Area V: Supporting active participation in the community}

Skills:

1. Supports community participation and contribution.

Tasks:

- Implements plans, as directed, to promote community connections.

- Supports community connections and activities through personal interest, 
- contribution and productivity.

- In an unbiased fashion, facilitates the opportunity for civic engagement, such as voting.

\section{Competency Area W: Supporting employment, educational, and career goal attainment}

Skills:

1. Supports the individual by being knowledgeable about the career and employment goals of the individual.

2. Supports the individual by being knowledgeable about the educational goals of the individual.

3. Develops and supports the individual's skills to help the individual meet the productivity expectations of the workplace.

Tasks:

- Implements plans, as directed, to support career and employment interests and goals of the individual.

- Implements plans, as directed, to support educational interests and goals of the individual.

- Can describe the educational interests of the individual.

- Can describe and discuss the educational supports needed by the individual.

- Follows the ISP for job skill development.

- Can describe to the individual the workplace expectations for productivity and conduct.

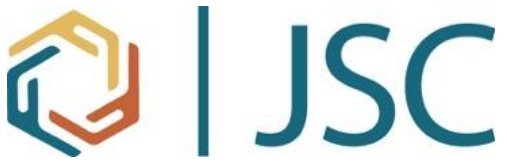

The Journal of Social Change, sponsored by Walden University, welcomes manuscripts focusing on interdisciplinary research in social change that improves the human condition and moves people, groups, organizations, cultures, and society toward a more positive future. 